\title{
News and Information
}

\section{WHO Global Database on Body Mass Index (BMI): an interactive surveillance tool for monitoring nutrition transition}

The WHO Global Database on Body Mass Index (BMI) is now available on line at http://www.who.int/bmi/index. jsp (directly) or http://www.who.int/nutrition/en/ (through the WHO Nutrition homepage).

The Database provides both national and sub-national adult underweight, overweight and obesity prevalence rates by country, year of survey and gender. The information is presented interactively as maps, tables, graphs and downloadable documents.

It was initiated as part of WHO's commitment to implementing the recommendations of the WHO Expert Consultation on Obesity: Preventing and Managing the Global Epidemic (Geneva, 3-5 June 1997), which identified the lack of nationally representative cross-sectional data as an obstacle for facilitating international comparisons of adulthood obesity rates, monitoring the magnitude of the current and future obesity problems, and evaluating the effectiveness of intervention strategies. During the last four years, the Database has evolved, in close collaboration with FAO, as a global interactive surveillance tool to monitor nutrition transition, covering and reporting on the entire spectrum of adult nutritional status.

This unique surveillance system also incorporates food availability data from the FAOSTAT Database, thereby providing a valuable contribution to an ongoing interagency work on monitoring food insecurity and vulnerability which was developed as follow-up to the World Food Summit (Rome, 1996). The Dietary Energy Supply (DES) data are displayed in conjunction with the BMI data on the maps and in the charts. DES figures are produced by FAO based on Food Balance Sheets (FBS).

The further development of the Database is underway. These include:

- Enhancement of underweight data.

- Addition of global and regional estimates.

- Further development of food consumption data.

- Incorporation of data for school-aged children and adolescents as part of the preparatory work for developing a growth reference for these age groups.

Contact address:

World Health Organization

Department of Nutrition for Health and Development (NHD)

The WHO Global Database on BMI
20 Avenue Appia

CH-1211 Geneva 27, Switzerland

Fax: +41227914156

Email: bmidata@who.int

DOI: 10.1079/PHN2006967

\section{Nutrition-Friendly Schools Initiative (NFSI)}

Nutrition-related health problems in children are increasingly significant causes of disability and premature death worldwide. While undernutrition continues to be a major problem in many developing countries, the problem of overweight and obesity have reached epidemic proportions globally, and both developed and developing countries are seriously affected. In some countries, the epidemic of obesity sits alongside continuing problems of undernutrition, creating a double burden of nutritionrelated ill-health among the population, including children.

Based on the principle that effectively addressing the increasing global public health problem of the double burden of nutrition-related ill-health requires common policy options, the Nutrition-Friendly Schools Initiative (NFSI) has been developed as follow-up to the WHO Expert Meeting on Childhood Obesity (Kobe, 20-24 June 2005).

The main aim of the NFSI is to provide a framework for designing integrated school-based intervention programmes which address the double burden of nutritionrelated ill-health, building on and interconnecting the ongoing work of various agencies and partners. These include the FRESH Initiative, Essential Package (UNICEF/WFP), Child-Friendly Schools (UNICEF), Health Promoting Schools (WHO), School Food and Nutrition Education programmes (FAO) to mention just a few. NFSI applies the concept and principles of the Baby-friendly Hospital Initiative (BFHI).

Improving the nutritional status of school-aged children is an effective investment for the future generation. Preschools and schools offer many opportunities to promote healthy dietary and physical activity patterns for children, and are also a potential access point for engaging parents and community members in preventing child malnutrition in all its forms (i.e. undernutrition, micronutrient deficiencies, and obesity and other nutrition-related chronic diseases). The universality of the school setting for gaining access to children makes it highly relevant to global efforts to combat the increasing public health problems of the double burden of nutrition-related ill-health. 
Contact address:

World Health Organization

Department of Nutrition for Health and Development (NHD)

Nutrition-Friendly Schools Initiative (NFSI)

20 Avenue Appia

CH-1211 Geneva 27, Switzerland

Fax: +41227914156

DOI: $10.1079 /$ PHN2006968

\section{International Malnutrition Task Force of the International Union of Nutritional Sciences}

The International Union of Nutritional Sciences (IUNS) launched the Malnutrition Task Force at the IUNS Congress in Durban in September 2005. The initiative is supported by the International Pediatric Association (IPA), WHO, UNICEF ${ }^{1}$.

Malnutrition (underweight) contributes to approximately $60 \%$ of all child deaths. Despite the obvious impact of malnutrition on global health it does not capture the attention of policy-makers and lacks appropriate resources.

The WHO has developed guidelines for the treatment of severe malnutrition and a training course for doctors and nurses in which 10 steps are identified for routine case management, which can be effectively applied in resourcepoor settings. When properly implemented these guidelines are associated with reductions in case fatality (for example, from 22 to $7 \%$ in India ${ }^{2} ; 17$ to $4 \%$ in Bangladesh ${ }^{3,4} ; 34$ to $16 \%$ in Brazil ${ }^{5}$; 55 to $16 \%$ in Malawi ${ }^{6}$; further improvements are likely in Brazil and Malawi with fuller implementation).

There is thus clear evidence of benefit (health and cost savings). This experience needs to be shared and implemented as widely as possible. Serious life-threatening malnutrition evolves over time. The condition can be prevented and larger numbers can be treated effectively at an earlier stage of the disease process through active casefinding and community-based therapeutic care ${ }^{7}$. The two approaches of treatment, facility-based care and community-based care, are complementary. It is only if these relatively low-cost, evidence-based approaches are rolled out as standard better practice will it be possible to make a substantial impact, thereby making a contribution to the Millennium Development Goal of reducing child mortality by two-thirds.

\section{Aim of the Task Force}

With the aim of substantially reducing child malnutrition and case fatality, the Malnutrition Task Force has two immediate aims. Firstly, to ensure that an integrated system of prevention and treatment of malnutrition is actively supported as a fundamental aspect of care, and becomes an integral part of all training programmes for all health workers, including doctors, nurses and public health nutritionists. Secondly, to work towards capacity building and the strengthening of health systems for early identification and treatment at home.

In the first instance the activities of the Task Force will focus on how best to build capacity and to develop advocacy.

- For building capacity:

- Establish three regional networks (South and Southeast Asia, sub-Saharan Africa, Latin America) to coordinate expertise and develop capacity building partnerships;

- Provide appropriate training and protocols for best practice;

- Encourage operational research to monitor and improve health workers' performance and to provide evidence of effect;

- Scale up activities.

- For advocacy:

- Raise the profile of malnutrition among makers of health policy and donor agencies;

- Dissemination of experience.

Therefore, for both there will be an urgent and immediate need to effectively mobilise resources to support expansion of programmes.

\section{Programme of activities}

IUNS has established a management structure that consists of a Board of Governors, chaired by the IUNS President, Professor Ricardo Uauy, and with representation from IPA, WHO, UNICEF. A Steering Committee convened by Ann Ashworth and Alan Jackson has regional representation and experts in both facility-based and community-based therapeutic care. An advisory panel of those with special expertise and experience is being drawn together, to best utilise the wide knowledge and skills of a range of health professionals in working towards the common purpose. Formal partnerships are being created with representatives of specific professional groups to create a framework within which the relevant policy decisions and objectives might be effectively realised.

In the past nutrition has been criticised for being fragmented and failing to act with clear purpose. Malnutrition remains a substantial scourge, for which we know the causes and their interaction, from the immediate personal factors up to the broader considerations in society. There is a challenge to every health professional in dealing with this problem effectively. Severely malnourished individuals deserve the straightforward approaches to life-saving care which have been clearly demonstrated. Those at immediate risk are entitled to be cared for at an early stage to ensure they do not slip imperceptibly to become a more difficult problem for treatment. Household 